\title{
Hierarchical Hypothesis and Feature-Based Blind Modulation Classification for Linearly Modulated Signals
}

\author{
Sudhan Majhi, Senior Member, IEEE, Rahul Gupta ${ }^{(0)}$, Weidong Xiang, Member, IEEE, \\ and Savo Glisic, Senior Member, IEEE
}

\begin{abstract}
This paper presents a hierarchical hypothesis test and a feature-based blind modulation classification (BMC) algorithm for linearly modulated signals. The proposed BMC method is based on the combination of elementary cumulant (EC) and cyclic cumulants. The EC is used to decide whether the constellations are from real, circular, or rectangular class, which is referred to as macro classifier. The cyclic cumulant is used to classify modulation within a subclass, which is referred to as micro classifier. For the micro classification, we use positions of nonzero cyclic frequencies (symbol rate frequency or carrier frequency) of the received signals. A hierarchical hypothesis-based theoretical framework has been developed to find the probability of error for the proposed classification. The method works over a flat fading channel without any knowledge of the signal parameters. The proposed method is more robust than the one based on EC and at the same time it requires lower complexity than the maximum likelihood approach. To validate the proposed scheme, measurement is carried out in realistic scenarios. The performance of the new algorithm is compared with the existing methods. In this paper, we have considered a six-class problem including binary phase-shift keying, quadrature phase-shift keying (QPSK), offset-QPSK, $\pi / 4$-QPSK, minimum shift keying, and 16-quadrature amplitude modulation.
\end{abstract}

Index Terms-Blind modulation classification, cyclic cumulants, elementary cumulant, testbed implementation.

\section{INTRODUCTION}

B LIND modulation classification (BMC) is a rapidly evolving area with applications in monitoring, spectrum management in cognitive radio, and cooperative communications systems [1], [2]. The blind estimation process is bandwidth efficient as it is non-data aided and does not use the predefined training sequence or pilot symbols in the estimation process. As a result, classifier processing time gets reduced. Bandwidth

This work was partially supported by DST, India. The review of this paper was coordinated by Dr. C. Yuen. (Corresponding author: Rahul Gupta.)

S. Majhi and R. Gupta are with the Department of Electrical Engineering, Indian Institute of Technology Patna, Patna 801103, India (e-mail: smajhi@ iitp.ac.in; rahul.pee15@iitp.ac.in).

W. Xiang is with the Department of Electrical and Computer Engineering, University of Michigan, Dearborn, MA 48128 USA (e-mail: xwd@umich.edu).

S. Glisic is with the Centre for Wireless Communication, University of Oulu, Oulu 90550, Finland (e-mail: savo.glisic@ee.oulu.fi). efficient transmission not only increases the effective data-rate of the system, but also cuts down the cost of spectrum licensing [3]. Moreover, the training sequence based approaches do not perform well in the presence of synchronization errors like carrier frequency offset, phase jitters, independent and identically distributed (iid) fading channel assumption as well as severe channel conditions where the training sequences may lose their correlation properties completely [4]. The BMC facilitates adaptive system and dynamic data rate transmission [5]-[8].

In the recent years, with the emergence of new communication technology interest in BMC algorithms has been increased considerably. BMC is a sensitive part in the demodulation process, since the classifier success rate has to be very high for a truthful communication. However, the conventional BMC approaches are classifying modulation schemes based on the knowledge of the signal parameters such as carrier frequency, symbol rate, carrier phase, frequency offset and timing offset. For instance, although the coherent maximum likelihood (ML) algorithm has very good performance, it requires knowledge of all the signal parameters to start the modulation classification process [9]-[11]. There are diverse methods on BMC in the literature based on unknown signal parameters as well [12], [13]. However, most of the known methods require knowledge of signal parameters for recognizing modulation format. On the other hand, the knowledge of modulation schemes in advance may be essential to perform estimation of the other parameters.

Several BMC algorithms such as ML, pattern reorganization, minimum distance centroid estimator, non-parametric likelihood function, 2-D histograms, and probability distance approach have been published in the literature [14]-[19]. The ML method needs very high computational complexity and is very sensitive to the symbol timing offset, carrier frequency offset and fading channels [9]-[11]. Particularly, it requires data acquisition, data representation, and recognition. Storage requirements for 2-D histograms are very high and estimating 2-D probability density functions (pdfs) is not an easy task.

Recently, feature based statistical methods have been found to be more useful. The BMC algorithm based on a higher order statistical moment, elementary cumulant (EC), cyclic cumulant and expectation-maximization have been studied in detail in [20]-[24]. The hierarchical EC approach presented in [20] has a good performance over AWGN. The higher order cumulant 
methods discussed in [23], [24] perform well even in the fading channel. But the ECs are not able to differentiate between circular constellation modulation formats, i.e., quadrature phaseshift keying (QPSK), offset-QPSK (OQPSK), $\pi / 4-\mathrm{QPSK}$ and minimum shift keying (MSK). The cyclic cumulant approach and their combined methods [25]-[29] are robust to various impairments, such as synchronization errors like timing offset, carrier frequency offset, phase jitters and have a satisfactory performance. However, cyclic cumulants are not able to differentiate between binary phase-shift keying (BPSK), QPSK, OQPSK, 16-quadrature amplitude modulation (16QAM) since for all four modulation schemes the fourth order cyclic cumulant peak appears at $4 w_{c}$. To the best of our knowledge, no modulation classifier incorporates these six-class problems, especially circular constellations modulation format.

To address the above problems, a new BMC algorithm is proposed, implemented and experimented over a practical scenarios by using National Instrument (NI) testbed [30], [31]. The proposed BMC algorithm is based on the combination of the fourth order EC as well as the fourth and the second order cyclic cumulants. Simulation, testbed implementation and measurement are carried out based on the six-class problem which includes BPSK, QPSK, OQPSK, $\pi / 4$-QPSK, MSK, and 16-QAM schemes. These modulations are widely used in conventional communication systems. A hierarchical hypothesis model has been developed for theoretical performance analysis. It is observed from the theoretical and measurement studies that the proposed hierarchical hypothesis and feature-based BMC algorithm adds robustness against various impairments such as carrier frequency offsets, phase offset, timing offset and works well even in a flat fading channel.

The rest of the paper is organized as follows. Section II discusses a signal model of discrete cyclostationary passband signal and discrete cyclostationary baseband signal. Section III presents BMC algorithm based on macro and micro classifications. In this section, theoretical derivations and hierarchical classification algorithm are discussed. Section IV describes implementation and measurement setup of the testbed. Section V illustrates simulation and measurement results and Section VI concludes the paper. Finally, the fourth and the second order cumulant pdfs are derived in Appendix.

\section{Signal Model}

In this study, we assume that signal is a cyclostationary process (CP). In fact, most of the man-made signals are cyclostationary. We use an intermediate frequency (IF) signal and complex baseband signal at the receiver for both macro and micro classifications. A digitized IF signal is obtained by down conversion of the received RF signal and the baseband signal is obtained by an additional down-conversion and filtering of the digitized IF signal. The IF frequency is determined by a coarse and fine carrier frequency estimations. The coarse carrier frequency estimate is obtained by smoothing power spectral density (PSD) of the received IF signal. The fine carrier frequency estimate is obtained by using fourth order cyclic cumulant proposed in [2], [32].
In this context, we consider a real discrete-time cyclostationary signal. Let us start with a continuous-time domain, where the transmitted signal is given by

$$
x(t)=\operatorname{Re}\left(\sum_{l=0}^{L-1} a[l] \tilde{g}(t-l T-\tau) e^{-j\left(w_{c} t+\phi\right)}\right) .
$$

In (1) $L$ is the number of symbols, $a[l]$ the $l$ th symbol drawn from any of six-class modulation schemes, $T$ the symbol period, $\tau$ the timing offset, $w_{c}$ the carrier frequency, ${ }^{1}$ and $\phi$ the carrier phase noise. The transmitted pulse shape is $\tilde{g}(t)$ representing the root raised cosine (RRC) function, with roll-off factor $\beta$, $0 \leq \beta \leq 1$. Eq (1) can be deduced as

$$
x(t)=s_{r}(t) \cos \left(w_{c} t+\phi\right)+s_{i}(t) \sin \left(w_{c} t+\phi\right),
$$

where $a[l]=a_{r}[l]+j a_{i}[l], s_{r}(t)=\sum_{l=0}^{L-1} a_{r}[l] \tilde{g}(t-l T-\tau)$, $s_{i}(t)=\sum_{l=0}^{L-1} a_{i}(l) \tilde{g}\left(t-l T-\tau+\tau_{1}\right)$ and,

$$
\tau_{1}= \begin{cases}T / 2, & \text { OQPSK, MSK } \\ 0, & \text { otherwise }\end{cases}
$$

The signal received over the channel with Rayleigh fading and AWGN can be written as

$$
\tilde{y}(t)=h x(t)+v(t)
$$

In (4) $v(t)$ is the complex AWGN with two sided PSD with variance, $\sigma_{v}^{2}=N_{0} / 2$ where, $N_{0}$ is PSD of noise. The signal $\tilde{y}(t)$ is over sampled at rate of $P / T$ to yield the discrete-time version

$$
\begin{aligned}
\tilde{y}[n] & =\operatorname{Re}\left(\sum_{l=0}^{L-1} a[l] \tilde{g}\left[n T_{s}-l P T_{s}-N_{1} T_{s}\right] e^{-j w_{c} n T_{s} \phi}+v\left[n T_{s}\right]\right) \\
& =\operatorname{Re}\left(\sum_{l=0}^{L-1} a[l] \tilde{g}\left[n-l P-N_{1}\right] e^{-j w_{c} n+\phi}+v[n]\right)
\end{aligned}
$$

This is a cyclostationary signal for oversampling factor $P>4$ [33], where $T_{s}$ is the sampling instant and $N_{1}$ is the number of samples corresponding to the delay $\tau$. The oversampling factor is defined as $P=F_{s} / f_{s}=T / T_{s}=N / L$ where $F_{s}$ denotes the sampling rate, $f_{s}=1 / T$ the symbol rate, $N$ the received signal length, and $T_{s}=1 / F_{s}$ the sampling period which is small enough for all possible transmitted bit rates. The resultant oversampled signal is free from inter-symbol interference. The above real signal can be represented in terms of complex signal as

$$
\begin{aligned}
y[n] & =\tilde{y}[n]+j \hat{\tilde{y}}[n] \\
& =\left(s_{r}[n]-j s_{i}[n]\right) e^{j w_{c} n+\phi}+v[n] \\
& =s[n] e^{j\left(w_{c} n+\phi\right)}+v[n],
\end{aligned}
$$

where $\hat{\tilde{y}}[n]$ is a Hilbert transform of $\tilde{y}[n]$.

The problem statement can be formulated as follows: Given $N$ samples, $\{y[n]\}_{1}^{N}$, IF carrier frequency and modulation format needs to be estimated for six linearly modulated signals without having any prior knowledge of the signal parameters,

\footnotetext{
${ }^{1}$ We refer carrier frequency $w_{c}$ as a frequency of a IF signal
} 
TABLE I

TheOritical Value of Fourth ORDER Elementary Cumulant $\hat{c}_{[s, 4,2]}$

\begin{tabular}{lccccc}
\hline \hline BPSK & QPSK & OQPSK & $\pi / 4 Q P S K$ & MSK & 16QAM \\
\hline-2 & -1 & -1 & -1 & -1 & -0.68 \\
\hline \hline
\end{tabular}

$\left\{a[l], g[n], \beta, N_{1}, f_{o}, \phi, L\right\}$. The carrier IF frequency estimator and modulation classifier need only a short burst of the received signals, i.e., only 500 symbols are sufficient for successful blind estimation and blind classification. Thus, between two successful modulation classifications, $500 T_{s}$ time is required. In other words, if the transmitter needs to change modulation scheme adaptively, the minimum time required from changing one modulation to another is $500 T_{s}$.

Once the carrier frequency of IF signal is retrieved a baseband signal can be obtained as

$$
s[n]=\sum_{l} a[l] \tilde{g}\left[n-l P-N_{1}\right] e^{-j\left(2 \pi f_{o} n\right)}+w[n],
$$

where $w[n]$ is the low-pass complex baseband noise. We assume that the frequency offset $f_{o}$, in the above signal is about $\pm 100 \mathrm{~Hz}$ and can be neglected. It has been observed that after the estimation of IF carrier, a negligible frequency offset, $\left|f_{o}\right| \leq \pm 100 \mathrm{~Hz}$, present in the received baseband signals, does not affect the modulation classification.

\section{Modulation Classification}

A considerable research work has been published on BMC by using EC [20]. The statistical tool, EC, has strong properties to classify modulation from one subclass to another. EC classifies modulation formats between BPSK/PAM and QPSK or between M-PSK and M-QAM. However, it is unable to classify modulation format within the subclass which contains signals from a circular constellation point. As an example, the modulation QPSK, OQPSK, $\pi / 4-$ QPSK and MSK have the same EC values illustrated in Table I. On the other hand, cyclic cumulant provides good approach to classify modulation within the subgroup. However, it has inferior performance while classifying modulation format from different subgroups.

\section{A. Macro Modulation Classification}

It has been seen from [20] that EC approach, as macro classification technique is an efficient way to classify modulation schemes from one subclass to another over an AWGN channel. We extend this macro modulation classification technique for a flat fading channel. As the macro modulation classification is performed immediately after the IF carrier frequency estimation, we can assume that a complex baseband signal is available to the macro modulation classifier. The complex baseband discretetime signal represented as in (7). CP can be viewed as multiple interleaved stationary processes (SP). Thus, in wide-sense, $\mathrm{CP}$ is a SP. For a complex-valued stationary random process $s[n]$, second-order EC can be defined in two different ways depending on placement of conjugation given as [20]

$$
\begin{aligned}
& c_{[s, 2,0]}=E\{s[n] s[n]\} \\
& c_{[s, 2,1]}=E\left\{s[n] s^{*}[n]\right\} .
\end{aligned}
$$

In $c_{[s, 2,0]}$ and $c_{[s, 2,1]}$, the first subscript represents the baseband signal, second subscript represents the order of EC, and third subscript represent the number of conjugates used in the operation. Similarly, the fourth-order EC with two-conjugate can be written as

$$
c_{[s, 4,2]}=\operatorname{cum}\left(s[n], s[n], s^{*}[n], s^{*}[n]\right) .
$$

The sampled estimates of (8) and (9) are given by

$$
\begin{aligned}
& \hat{c}_{[s, 2,0]}=\frac{1}{N} \sum_{n=1}^{N} s[n]^{2}, \\
& \hat{c}_{[s, 2,1]}=\frac{1}{N} \sum_{n=1}^{N}|s[n]|^{2},
\end{aligned}
$$

and

$$
\hat{c}_{[s, 4,2]}=\frac{1}{N} \sum_{n=1}^{N}|s[n]|^{4}-\left|\hat{c}_{[s, 2,0]}\right|^{2}-2 \hat{c}_{[s, 2,1]}^{2},
$$

where the superscript ${ }^{\wedge}$ denotes a sample average. It is noted that the above EC has the form of the sum of signal and noise. In practice, we estimate the normalized EC by

$$
\tilde{c}_{[s, 4,2]}=\hat{c}_{[s, 4,2]} / \tilde{c}_{[s, 2,1]}^{2},
$$

where $\tilde{c}_{[s, 2,1]}=\hat{c}_{[s, 2,1]}-\hat{c}_{[s, 2,1, w]}$ and $\hat{c}_{[s, 2,1, w]}$ is an estimated variance of AWGN, $w[n]$. In the implementation, we estimate $\tilde{c}_{[s, 2,1]}$ from a receiver system noise floor through a calibration process.

The macro modulation classification is based on the threshold detection as suggested in [20]. In practice, we calculate thresholds, feature values, for different modulated signals from histograms of their moments and ECs. Table I shown below presents the theoretical values of fourth order EC [20], [29]. These values are obtained for equiprobable samples under noise free and no fading assumptions, for different modulation schemes. In general, these assumptions are not realistic, so we have considered 200-500 samples and performed extensive simulation under flat fading conditions where real experimental data at different SNR have been tested. After that we have selected a range of thresholds shown in Fig. 1, based on the feature values of fourth order EC. The threshold value is independent above $4 \mathrm{~dB}$ SNR and above certain number of samples (200-500). Below 4 dB SNR and below two hundred samples the variance of the estimated fourth order EC is wider, resulting in degraded classification performance which is one of the limitation of the proposed algorithm. However, we have performed the experiment with this threshold at different measurement setup at different places, and the algorithm still works. In practice, the threshold can be determined easily through a system calibration process.

The macro modulation classification may fail over severe channel condition. In this work, we have considered a Rayleigh 


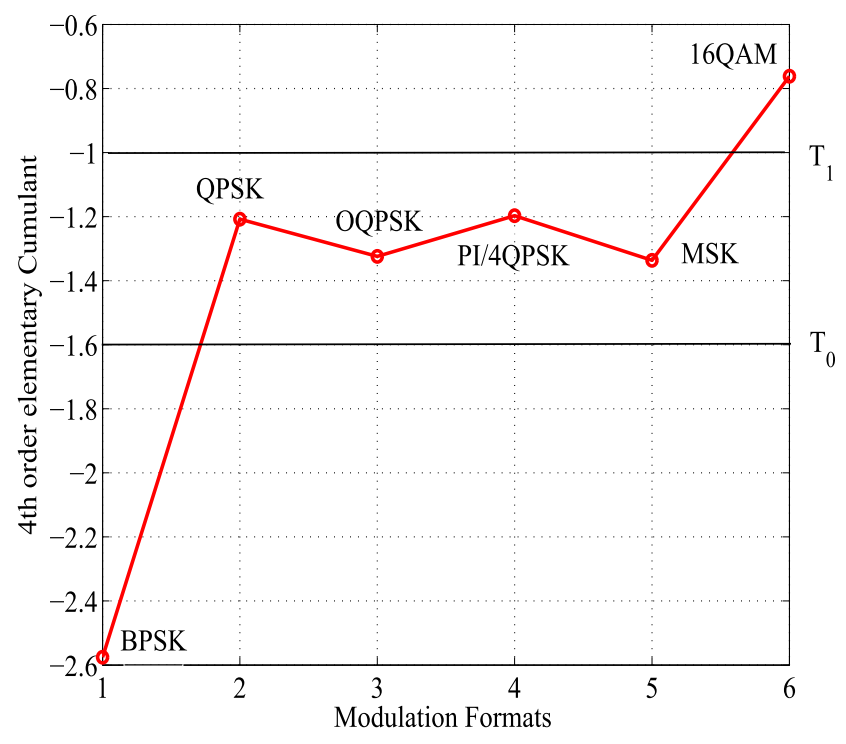

Fig. 1. Macro modulation classification for six modulation formats by fourth order elementary cumulant in a Rayleigh flat fading channel.

flat fading channel. It is worth to mention that the macro modulation classification is very sensitive to phase noise and frequency offset. The presence of phase noise mainly makes it uncertain to classify signals with circular constellation points, thus we do not use this macro classifier to classify these types of modulated signals. The presence of larger frequency offset is ruled out by perfect estimation of IF frequency described in Section II. Thus, we can easily distinguish BPSK and 16-QAM as shown in Fig. 1 by defining threshold values $\hat{c}_{[s, 4,2]}<T_{0}, \hat{c}_{[s, 4,2]}>T_{1}$ for BPSK and 16-QAM modulated signal respectively. It is observed that the macro classification technique cannot classify QPSK, OQPSK, $\pi / 4-Q P S K$, and MSK modulation format as they have almost the same EC values between $T_{0} \leq \hat{c}_{[s, 4,2]} \leq T_{1}$. It is mainly due to the presence of phase noise described above.

\section{B. Micro Modulation Classification}

The BMC algorithm in [20] showed adequate performance over an AWGN channel. However, the algorithm becomes inadequate for a Rayleigh flat fading channel, especially when modulated signals are from the circular constellations. We use a novel approach for micro modulation classification based on the fourth order cyclic cumulant of the IF signals and the second order cyclic cumulant of the baseband signal. The features of the cyclic cumulants for different modulation formats are studied below:

1) The fourth Order Cyclic Cumulant: The fourth order time-varying correlation function of $y[n]$ at time lag $\mathbf{0}=[0,0,0]$ can be expressed as [32]

$$
c_{r[y, 4,0]}[n ; \mathbf{0}]=\sum_{l=1}^{L} \sigma_{s_{l}}^{4} e^{4 j\left(w_{c} n+\phi\right)}+\sigma_{v}^{4},
$$

where $\sigma_{s_{l}}^{4}=E\left\{s_{l}^{4}[n]\right\}$, and $\sigma_{v}^{4}=E\left\{v^{4}[n]\right\}$. The corresponding Fourier series (FS) coefficient of $c_{r[y, 4,0]}[n ; \mathbf{0}]$, known as cyclic

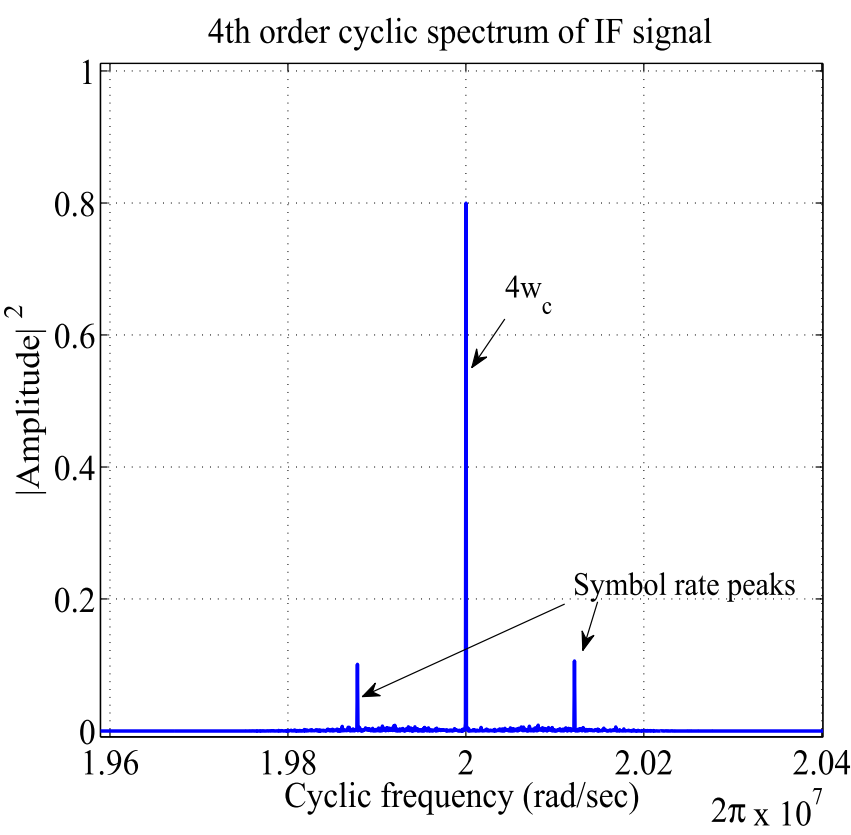

Fig. 2. Non-zero cyclic cumulant peak for QPSK modulated signals appears at four times of IF carrier frequency and at $4 w_{c} \pm w_{s}$ frequencies where $w_{s}$ is the symbol rate frequency.

cumulant, can be expressed as

$$
C_{[y, 4,0]}[\alpha ; \mathbf{0}]=\sum_{l=1}^{L} \sigma_{s_{l}}^{4} e^{j \phi} \delta\left[\alpha-4 w_{c}\right]+\sigma_{v}^{4} \delta[\alpha],
$$

where $\alpha$ is cyclic frequency. In practice, (14) can be estimated as [32]

$$
\hat{C}_{[y, 4,0]}[\alpha ; \mathbf{0}]=\frac{1}{N} \sum_{n=0}^{N-1} y^{4}[n] e^{-j n \alpha} .
$$

Note that in fact (15) is nothing but the normalized discrete Fourier transform (DFT) of $y^{4}[n]$, and can be implemented efficiently using the fast Fourier transform (FFT) algorithm. Once $\hat{C}_{[y, 4,0]}[\alpha ; \mathbf{0}]$ is obtained, frequency estimation is straightforward, given in [2], [32]

$$
\hat{w}=\underset{\alpha \in\left[\bar{\gamma}_{3}, \bar{\gamma}_{4}\right]}{\arg \max }\left|\hat{C}_{[y, 4,0]}[\alpha ; \mathbf{0}]\right|,
$$

where $\left[\bar{\gamma}_{3}, \bar{\gamma}_{4}\right]$ is the interval discussed in Section III-C.

The non-zero cyclic cumulant peaks for QPSK and OQPSK modulated signal appear at $4 w_{c}$ in Figs. 2 and 3 as an instantaneous value, but for $\pi / 4-\mathrm{QPSK}$ and MSK modulated signals, these appear at different positions. The construction of cyclic cumulant for $\pi / 4-$ QPSK and MSK modulated signals is a bit different than for QPSK and OQPSK. The fourth order cyclic cumulant for $\pi / 4-\mathrm{QPSK}$ and MSK modulated signals are described below:

a) Cyclic Cumulant Peakfor $\pi / 4-Q P S K$ : The transmitted signal for $\pi / 4-Q P S K$ can be expressed as [34]

$$
\begin{aligned}
S_{\pi / 4-Q P S K}(t) & =u_{k} \cos \left(w_{c} t\right)-v_{k} \sin \left(w_{c} t\right) \\
& =A \cos \left(w_{c} t+\phi_{k}\right),
\end{aligned}
$$




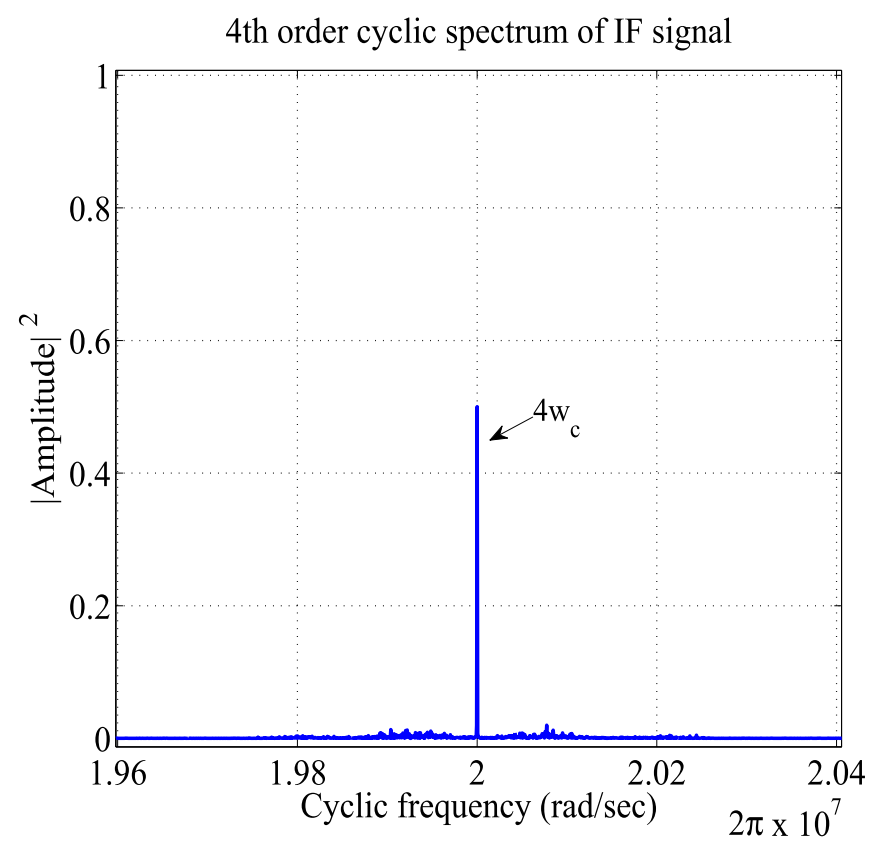

Fig. 3. Non-zero cyclic cumulant peak for OQPSK modulated signals appears at four times of IF carrier frequency. The symbol rate frequency is disappeared for OQPSK due to the presence of $T / 2$ offset between I and Q phase [1].

where $u_{k}$ and $v_{k}$ are the amplitudes in the $k t h$ symbol interval of coded I and Q signal component respectively, the signal amplitude is $A=\sqrt{u_{k}^{2}+v_{k}^{2}}$ and phase $\phi_{k}=\tan ^{-1} \frac{v_{k}}{u_{k}}$. The baseband representation of $\pi / 4-\mathrm{QPSK}$ modulated signal is $s_{p}(t)=A e^{j \phi_{k}}$. The phase $\phi_{k}$ is a differentialy encoded and can be expressed as $\phi_{k}=\phi_{k-1}+\Delta \phi_{k}$, where phase, $\Delta \phi_{k}= \pm \pi / 4, \pm 3 \pi / 4$, and in general it is written as

$$
\begin{aligned}
\Delta \phi_{k} & =\Delta w_{k} \cdot \Delta t_{k} \\
\pm \pi / 4 & =2 \pi f_{k} \cdot T_{s} \\
f_{k} & = \pm f_{s} / 8 .
\end{aligned}
$$

In (18) $\Delta w_{k}$ is the change of angular frequency, $f_{k}$ is the corresponding frequency and $\Delta t_{k}$ is the change of time in the kth symbol interval. The baseband signal for $\pi / 4-Q P S K$ can further be expressed as

$$
\begin{aligned}
s_{p}(t) & =A e^{j \phi_{k}}=A e^{j 2 \pi f_{k} t} \\
& =A e^{j 2 \pi \frac{ \pm f_{s}}{8} t} \\
& =A e^{j \frac{ \pm w s}{8} t},
\end{aligned}
$$

where, $w_{s}$ is angular symbol rate frequency, and the received IF signal of $\pi / 4-Q P S K$ can be given as

$$
\begin{aligned}
y_{p}(t) & =\sum_{l=1}^{L} s_{p_{l}}(t) e^{j\left(w_{c} t+\phi\right)}+v(t) \\
& =\sum_{l=1}^{L} A_{l} e^{j \frac{ \pm w s}{8} t} e^{j\left(w_{c} t+\phi\right)}+v(t),
\end{aligned}
$$

where $A_{l}$ is amplitude of the $l t h$ symbol, $s_{p_{l}}(t)$.
The signal $y_{p}(t)$ is over sampled at rate of $P / T$ to yield the discrete-time version $y_{p}[n]$. The fourth order time-varying correlation function of $y_{p}[n]$ at time lag $\mathbf{0}=[0,0,0]$ can be expressed as [32]

$$
c_{r\left[y_{p}, 4,0\right]}[n ; \mathbf{0}]=\sum_{l=1}^{L} \sigma_{l}^{4} e^{j \frac{ \pm w_{s}}{2} n} e^{4 j\left(w_{c} n+\phi\right)}+\sigma_{v}^{4},
$$

where $\sigma_{l}^{4}=E\left[A_{l}^{4}\right]$ and $\sigma_{v}^{4}=E\left[v^{4}(n)\right]$, and the generalized FS coefficient of $c_{r\left[y_{p}, 4,0\right]}[n ; \mathbf{0}]$ is given by

$$
\begin{aligned}
C_{\left[y_{p}, 4,0\right]}[\alpha ; \mathbf{0}]= & \lim _{N \rightarrow \infty} \frac{1}{N} \sum_{n=0}^{N-1} c_{r\left[y_{p}, 4,0\right]}[n ; \mathbf{0}] e^{-j \alpha n} \\
= & \sum_{l=1}^{L} \sigma_{l}^{4} e^{4 j \phi} \lim _{N \rightarrow \infty} \frac{1}{N} \sum_{n=0}^{N-1} e^{-j n\left(\alpha-\left(4 w_{c} \pm \frac{w s}{2}\right)\right)} \\
& +\lim _{N \rightarrow \infty} \frac{1}{N} \sum_{n=0}^{N-1} \sigma_{v}^{4} e^{-j \alpha n} .
\end{aligned}
$$

Eq (22) can be simplified as

$$
C_{\left[y_{p}, 4,0\right]}[\alpha ; \mathbf{0}]=\sum_{l=1}^{L} \sigma_{l}^{4} e^{4 j \phi} \delta\left(\alpha-\left(4 w_{c} \pm \frac{w_{s}}{2}\right)\right)+\sigma_{v}^{4} \delta(\alpha) .
$$

In practice, (23) can be estimated as

$$
\begin{aligned}
\hat{C}_{\left[y_{p}, 4,0\right]}[\alpha ; \mathbf{0}] & =\frac{1}{N} \sum_{n=0}^{N-1} y^{4}[n] e^{-j n \alpha} \\
\hat{w} & =\underset{\alpha \in\left[\hat{\gamma}_{2}, \hat{\gamma}_{3}\right] \text { or }\left[\hat{\gamma}_{4}, \hat{\gamma}_{5}\right]}{\arg \max }\left|\hat{C}_{\left[y_{p}, 4,0\right]}[\alpha ; \mathbf{0}]\right|,
\end{aligned}
$$

where $\left[\hat{\gamma}_{2}, \hat{\gamma}_{3}\right]$ and $\left[\hat{\gamma}_{4}, \hat{\gamma}_{5}\right]$ are the intervals discussed in Section III-C.

It can be seen that the non-zero cyclic cumulant frequency of IF signal of $\pi / 4-Q P S K$ appears at $4 w_{c} \pm w_{s} / 2$ which is shown in Fig. 4.

b) Cyclic Cumulant Peakfor MSK: The transmitted signal for MSK can be expressed as

$$
S_{M S K}(t)=\sum_{l=1}^{L} s_{m_{l}}(t) e^{\frac{j \pi t}{2 T_{b}}} \cdot e^{j\left(w_{c} t+\phi\right)},
$$

where symbol interval is $T=2 T_{b}$, bit rate $f_{b}=2 f_{s}, T_{b}$ bit duration, and $f_{s}$ the symbol rate. Baseband representation of MSK modulated signal is $s_{m}(t)=s(t) e^{\frac{j w s t}{2}}$, where $s(t)$ is the magnitude of I and Q phase components. The corresponding received signal for $\mathrm{MSK}$ is

$$
\begin{aligned}
y_{m}(t) & =\sum_{l=1}^{L} s_{m_{l}}(t) e^{j\left(w_{c} t+\phi\right)}+v(t) \\
& =\sum_{l=1}^{L} s_{l}(t) e^{\frac{j w_{s} t}{2}} e^{j\left(w_{c} t+\phi\right)}+v(t),
\end{aligned}
$$

where $s_{l}(t)$ is the magnitude of the $l t h$ symbol. After converting to the discrete-time version $y_{m}[n]$, the fourth order time-varying 


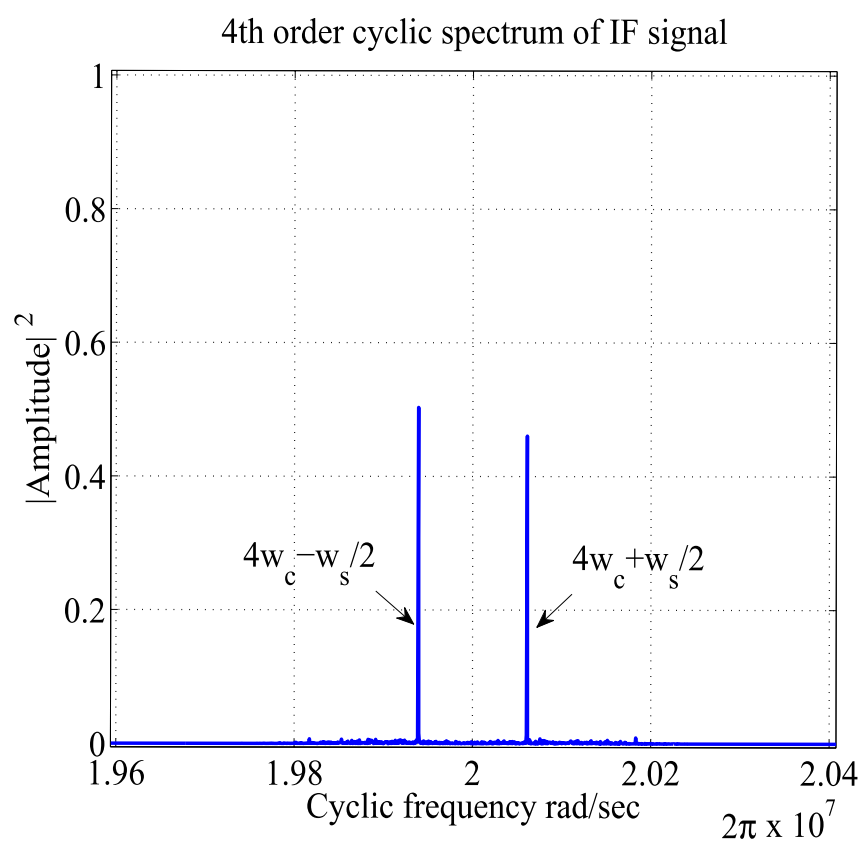

Fig. 4. Non-zero cyclic cumulant peak for $\pi / 4$-QPSK modulated signals appears at $4 w_{c} \pm \frac{w_{s}}{2}$.

correlation function of $y_{m}[n]$ at time lag $\mathbf{0}=[0,0,0]$ can be expressed as [32]

$$
c_{r\left[y_{m}, 4,0\right]}[n ; \mathbf{0}]=\sum_{l=1}^{L} \sigma_{m}^{4} e^{j 2 w_{s} n} e^{4 j\left(w_{c} n+\phi\right)}+\sigma_{v}^{4},
$$

where $\sigma_{m}^{4}=E\left[s_{l}^{4}(t)\right]$ and $\sigma_{v}^{4}=E\left[v^{4}(n)\right]$, and the generalized FS coefficient of $c_{r\left[y_{m}, 4,0\right]}[n ; \mathbf{0}]$ is given by

$$
\begin{aligned}
C_{\left[y_{m}, 4,0\right]}[\alpha ; \mathbf{0}]= & \lim _{N \rightarrow \infty} \frac{1}{N} \sum_{n=0}^{N-1} c_{r\left[y_{m}, 4,0\right]}[n ; \mathbf{0}] e^{-j \alpha n} \\
= & \sum_{l=1}^{L} \sigma_{m}^{4} e^{4 j \phi} \lim _{N \rightarrow \infty} \frac{1}{N} \sum_{n=0}^{N-1} e^{-j n\left(\alpha-\left(4 w_{c} \pm 2 w_{s}\right)\right.} \\
& +\lim _{N \rightarrow \infty} \frac{1}{N} \sum_{n=0}^{N-1} \sigma_{v}^{4} e^{-j \alpha n}
\end{aligned}
$$

Eq. (28) can be simplified as

$$
C_{\left[y_{m}, 4,0\right]}[\alpha ; \mathbf{0}]=\sum_{l=1}^{L} \sigma_{s}^{4} e^{4 j \phi} \delta\left(\alpha-\left(4 w_{c} \pm 2 w_{s}\right)+\sigma_{v}^{4} \delta(\alpha) .\right.
$$

In practice, (29) can be estimated as

$$
\begin{aligned}
\hat{C}_{\left[y_{m}, 4,0\right]}[\alpha ; \mathbf{0}] & =\frac{1}{N} \sum_{n=0}^{N-1} y^{4}[n] e^{-j n \alpha} \\
\hat{w} & =\underset{\alpha \in\left[\tilde{\gamma}_{1}, \tilde{\gamma}_{2}\right] \text { or }\left[\tilde{\gamma}_{5}, \tilde{\gamma}_{6}\right]}{\arg \max }\left|\hat{C}_{\left[y_{m}, 4,0\right]}[\alpha ; \mathbf{0}]\right|,
\end{aligned}
$$

where $\left[\tilde{\gamma}_{1}, \tilde{\gamma}_{2}\right]$ and $\left[\tilde{\gamma}_{5}, \tilde{\gamma}_{6}\right]$ are the intervals discussed in Section III-C.

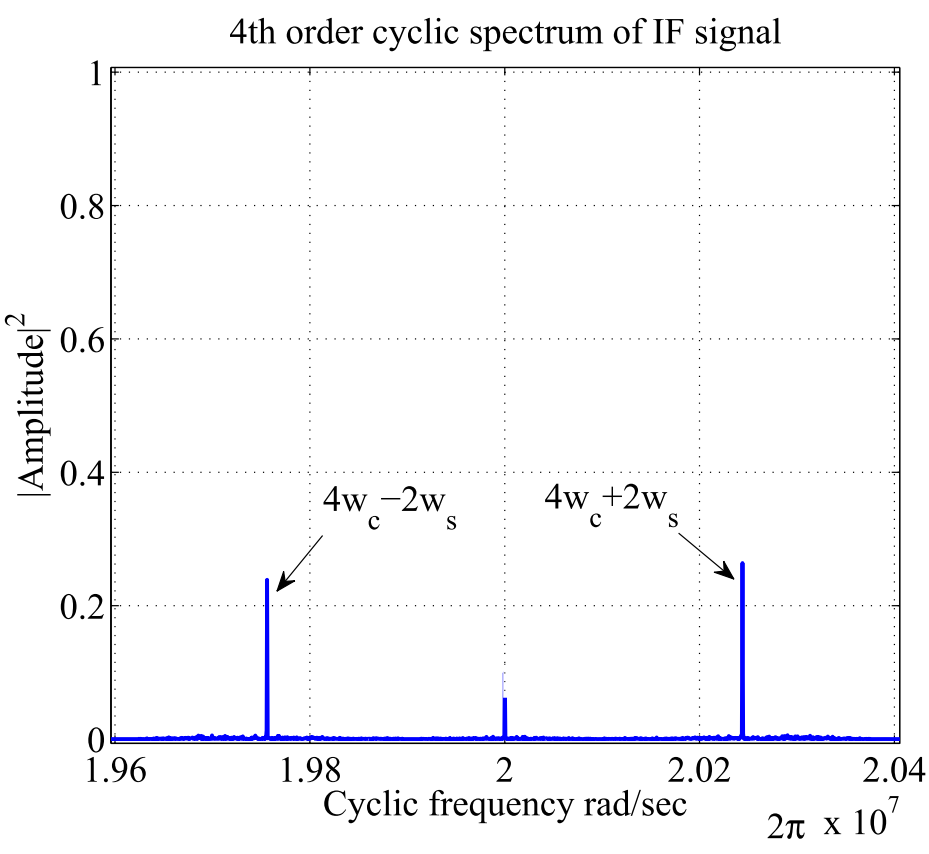

Fig. 5. Non-zero cyclic cumulant peak for MSK modulated signals appears at $4 w_{c} \pm 2 w_{s}$.

It can be seen that the non-zero cyclic cumulant frequency of IF signal for MSK appears at $4 w_{c} \pm 2 w_{s}$ which is shown in Fig. 5. Thus, QPSK, OQPSK, $\pi / 4-\mathrm{QPSK}$, and MSK have the distinct feature for their fourth order cyclic cumulant frequency. However, since QPSK and OQPSK provide non-zero cyclic cumulant frequency at the same position, these cannot be classified by using this feature.

\section{2) Second Order Cyclic Cumulant}

For classifying QPSK and OQPSK, we consider the second order EC of a complex baseband signal at zero time lag that can be expressed as in (8). To find out the periodicity of the energy of the signal, we have considered (8) as a time-varying correlation function for our implementation. The term $s[n] s^{*}[n]$ can be written as

$$
s[n] s^{*}[n]=\left|s_{r}[n]\right|^{2}+\left|s_{i}[n]\right|^{2}+|w[n]|^{2} .
$$

The I-component and Q-component of the the baseband signals are $s_{r}[n]=\sum_{l} a_{r}[l] g\left[n-l P-N_{1}\right]$ and $s_{i}[n]=$ $\sum_{l} a_{i}[l] g\left(n-l P-N_{1}\right]$ respectively. As $s[n]$ is a CP, the second order timing-varying correlation function of $s_{r}[n]$ with zero time lag can be expressed as [1].

$$
\begin{aligned}
c_{r\left[s_{r}, 2,1\right]}[n ; \mathbf{0}]= & \sum_{l} \sum_{m} E\left[a_{r}(l) a_{r}(m)\right] g\left(n-l P-N_{1}\right) \\
& \times g\left(n-m P-N_{1}\right) \\
= & \sigma_{r}^{2} \sum_{l} g^{2}\left(n-l P-N_{1}\right) \\
= & \frac{\sigma_{r}^{2}}{P} \sum_{l} G\left[\frac{l}{P}\right] e^{j 2 \pi n l / P} e^{-j 2 \pi l N_{1} / P}
\end{aligned}
$$




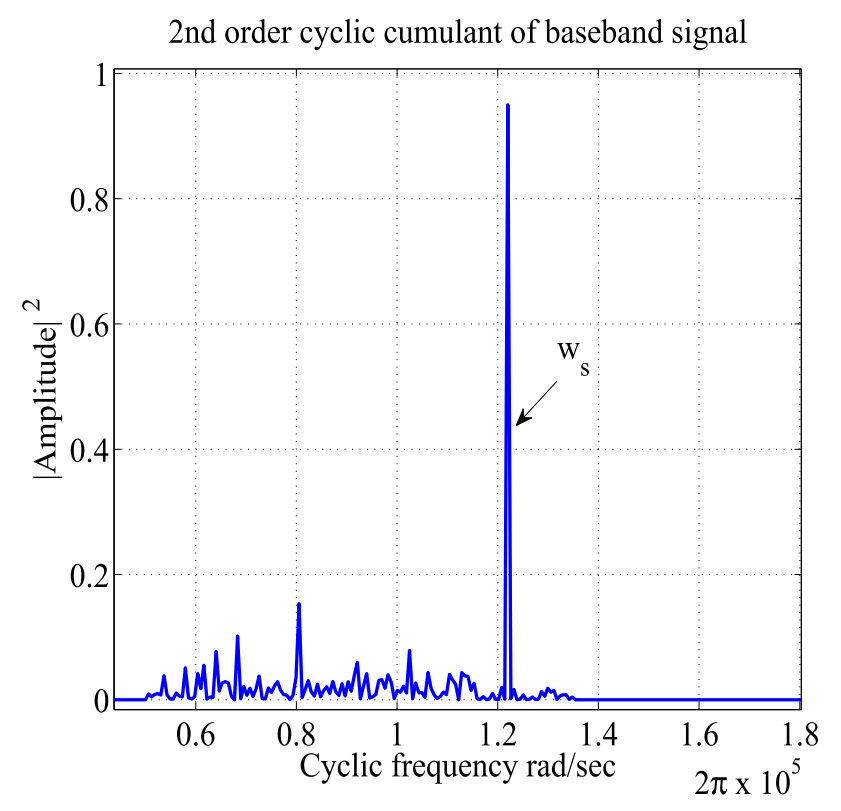

Fig. 6. Non-zero cyclic cumulant peak for the second order cyclic cumulant appears at $w_{s}$ of baseband QPSK modulated signals.

In (32) $\sigma_{r}^{2}=E\left[\left(a_{r}[l]\right)^{2}\right], G[$.$] is the DFT of g^{2}[$.$] , which is$ a raised cosine pulse and symmetric in nature. So, $G[$.$] is a$ real function, and (32) can be achieved by using poisson sum formula. Similarly, we can obtain the second order cyclic cumulant of $s_{i}[n]$. Now for $l=1$ the second order cyclic cumulant of $s[n]$ at cyclic frequency $\alpha$ can be obtained just by taking the FS,

$$
C_{[s, 2,1]}[\alpha ; \mathbf{0}]=\frac{\sigma_{a}^{2}}{P} G\left[\frac{1}{P}\right] \delta(\alpha-1 / P) e^{-j 2 \pi N_{1} / P}+\sigma_{w}^{2} \delta[\alpha],
$$

where $\sigma_{a}^{2}=\sigma_{r}^{2}+\sigma_{i}^{2}$. The non-zero cyclic cumulant frequency of baseband QPSK signal appears at symbol rate as shown in Fig. 6. In practice, (33) can simply be estimated as

$$
\begin{aligned}
\hat{C}_{[s, 2,1]}[\alpha ; \mathbf{0}] & =\frac{1}{N} \sum_{n=0}^{N-1} s^{2}[n] e^{-j n \alpha} \\
\hat{w} & =\underset{\alpha \in\left[\tilde{\gamma}_{7}, \tilde{\gamma}_{8}\right]}{\arg \max }\left|\hat{C}_{[s, 2,1]}[\alpha ; \mathbf{0}]\right|,
\end{aligned}
$$

where $\left[\tilde{\gamma}_{7}, \tilde{\gamma}_{8}\right]$ is interval discussed in Section III-C.

Eq. (33) can be applied for linearly modulated signals, such as BPSK, QPSK, 8-PSK and 16-QAM. However, for OQPSK, the second order cyclic cumulant does not provide non-zero cyclic peak at symbol rate frequency due to $\mathrm{P} / 2$ shift between I and Q components of OQPSK signal appearing in $s_{i}[n]=\sum_{l=0}^{L-1} a_{i}[l] g\left[n-l P-P / 2-N_{1}\right]$. The detailed derivation of second order cyclic cumulant of OQPSK is given in [1] as

$$
C_{[i, 2,1]}[\alpha ; \mathbf{0}]=\frac{\sigma_{i}^{2}}{P} \sum_{l} G\left[\frac{l}{P}\right] e^{-j 2 \pi l N_{1} / P} \delta\left[\alpha-\frac{l}{P}\right] e^{-j \pi l} .
$$

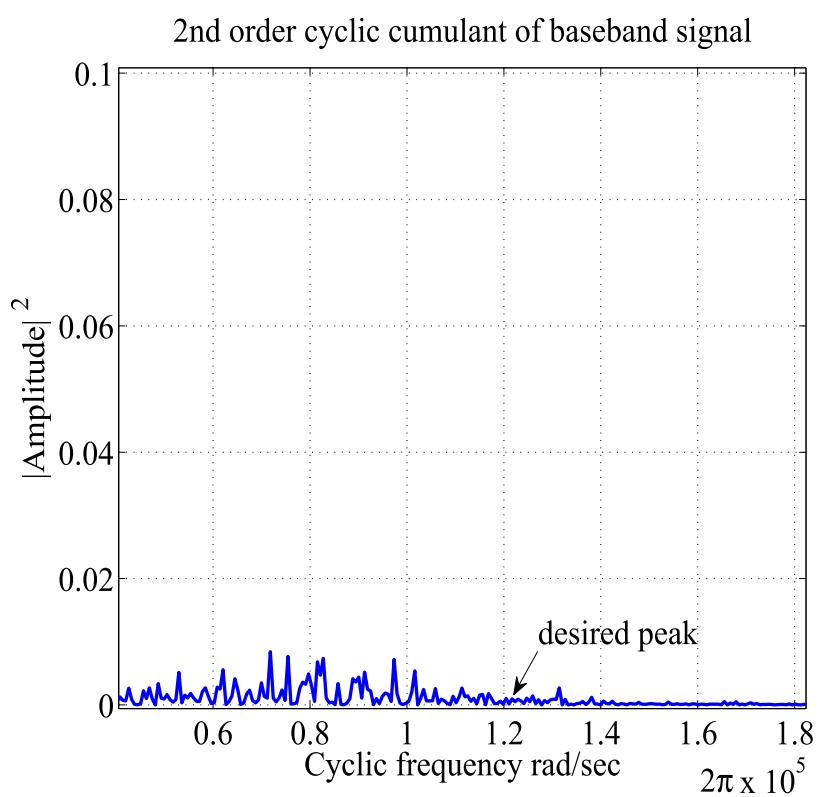

Fig. 7. Non-zero cyclic cumulant peaks for the second order cyclic cumulant of baseband OQPSK modulated signal, peaks seen in figure are due to noise present in the signal.

For $l=1$, we can find the second order cyclic cumulant for OQPSK as

$$
\begin{aligned}
C_{[s, 2,1]}[\alpha ; \mathbf{0}]= & \frac{1}{P} G\left[\frac{1}{P}\right] e^{-j 2 \pi N_{1} / P} \\
& \times\left(\sigma_{r}^{2} \delta\left[\alpha-\frac{1}{P}\right]-\sigma_{i}^{2} \delta\left[\alpha-\frac{1}{P}\right]\right)+\sigma_{w}^{2} \delta[\alpha] .
\end{aligned}
$$

In (36), cyclic spectrum function at $\alpha=1 / P$ is being canceled as $\mathrm{I}$ and $\mathrm{Q}$ component powers are the same, i.e., $\left.C_{[s, 2,1]}[\alpha ; \mathbf{0}]\right|_{\alpha=1 / P}=\sigma_{w}^{2} \delta(\alpha)$, and only noise term is left as shown in Fig. 7. At low SNR and for small roll-off factor, this approach may not be working, thus a weighted approach can be used provided in [1]. Therefore, a distinct feature between QPSK and OQPSK is observed from their second order cyclic cumulant.

\section{Hierarchical Hypothesis Classification Algorithm}

From Fig. 8 it is observed that the classification is a three stage hierarchical hypothesis based decision. For example, classification between hypothesis $\tilde{\mathcal{H}}_{231}$ and $\tilde{\mathcal{H}}_{232}$ i.e., QPSK and OQPSK depends on their previous classifications path $\tilde{\mathcal{H}}_{2} \rightarrow \tilde{\mathcal{H}}_{23}$. To find the overall performance of the classification, test statistics defined by (11), (15), (24) and (30) have to be derived from their respective hypothesis. For the received signal with different modulation schemes given as $\mathcal{H}_{i}: \tilde{y}_{i}[n]=h x_{i}[n]+v[n]$, and the corresponding baseband signal $s_{i}[n]=\tilde{x}_{i}[n]+w[n]$, where $n=0,1, \ldots, N-1, \mathcal{H}_{i}$ denotes the equiprobable hypothesis for six-class modulation schemes BPSK, MSK, $\pi / 4-\mathrm{QPSK}$, QPSK, OQPSK, and 16-QAM, i.e., $\{\underbrace{\mathcal{H}_{1}, \mathcal{H}_{2}, \mathcal{H}_{3}, \mathcal{H}_{4}, \mathcal{H}_{5}}_{\tilde{\mathcal{H}}_{1}}, \underbrace{\mathcal{H}_{6}}_{\tilde{\mathcal{H}}_{2}}\}$ 


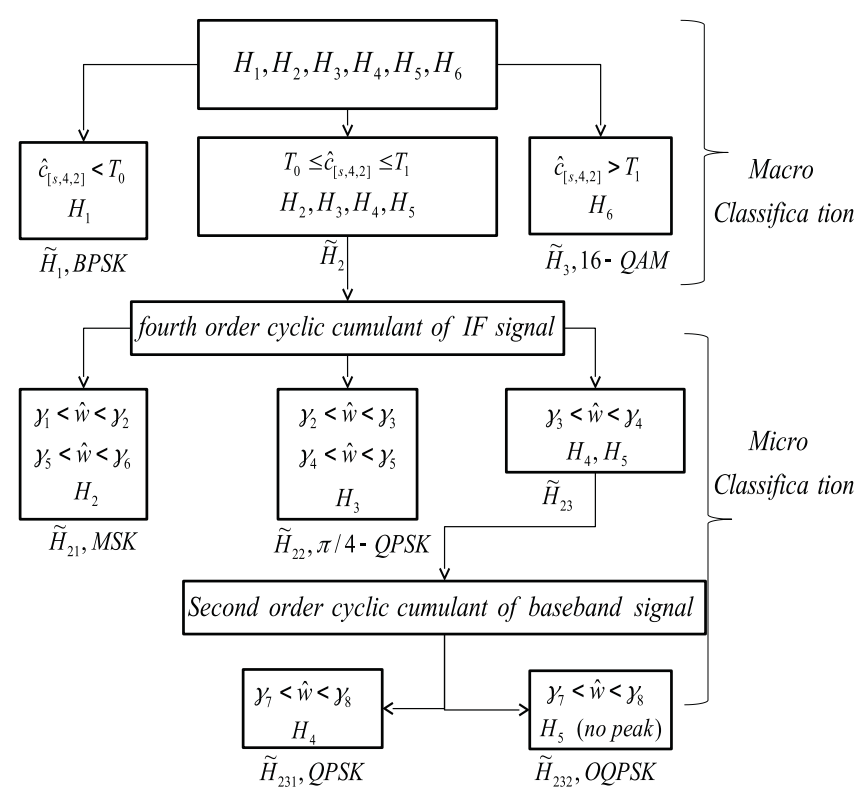

Fig. 8. Hierarchical hypothesis and feature-based blind modulation classification using elementary cumulants and cyclic cumulants.

respectively, where $\tilde{\mathcal{H}}_{1}, \tilde{\mathcal{H}}_{2}$ and $\tilde{\mathcal{H}}_{3}$ are the hypothesis in first stage of classification. The first stage of hierarchical classification uses the fourth order EC given by (11) which is a random variable and its instantaneous snap shot value is presented in Fig. 1, with the pdf of hypothesis given in Appendix.

So the first stage of classification classifies $\tilde{\mathcal{H}}_{1}, \tilde{\mathcal{H}}_{2}$ and $\tilde{\mathcal{H}}_{3}$ using the fourth order $\mathrm{EC}$ and the overall probability of correct classification $\left(P_{c c}\right)$ is given as

$$
\begin{aligned}
& P_{c c}= \sum_{i=1}^{3} p\left(\tilde{\mathcal{H}}_{i} \mid \tilde{\mathcal{H}}_{i}\right) p\left(\tilde{\mathcal{H}}_{i}\right) \\
&= \frac{1}{3} \sum_{i=1}^{3} p\left(\tilde{\mathcal{H}}_{i} \mid \tilde{\mathcal{H}}_{i}\right) \\
&= \frac{1}{3}\left[\begin{array}{c}
\operatorname{Pr}\left\{\hat{c}_{[s, 4,2]}<T_{0} \mid \tilde{\mathcal{H}}_{1}\right\} \\
\left(P_{1}\right)
\end{array}\right. \\
&+\operatorname{Pr}\left\{\begin{array}{c}
\left.T_{0}<\hat{c}_{[s, 4,2]}<T_{1} \mid \tilde{\mathcal{H}}_{2}\right\} \\
\left(P_{2}\right)
\end{array}\right. \\
&\left.+\operatorname{Pr}\left\{\hat{c}_{[s, 4,2]}>T_{1} \mid \tilde{\mathcal{H}}_{3}\right\}\right], \\
&\left(P_{3}\right)
\end{aligned}
$$

where $P_{1}, P_{2}, P_{3}$ are the probabilities of correct classification of real, circular, and rectangular constellation schemes.

The pdf of hypothesis for circular constellation signal is denoted by $p\left(\hat{w}\left|\mathcal{H}_{i}\right|_{i=2,3,4,5}\right)$ and the derivation is given in Appendix. Now the second stage of hierarchical classification can be applied to circular constellation signals MSK, $\pi / 4$-QPSK,

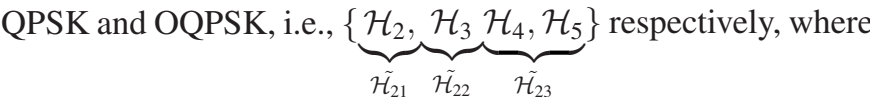
$\tilde{\mathcal{H}}_{21}, \tilde{\mathcal{H}}_{22}$ and $\tilde{\mathcal{H}}_{23}$ are the hypothesis in the second stage of classification. The probability of correct classification in the second stage hierarchical is given as

$$
\begin{aligned}
P_{2}= & \frac{1}{3}\left[\operatorname{Pr}\left\{\gamma_{1}<\hat{w}<\gamma_{2} \mid \tilde{\mathcal{H}}_{21}\right\}+\operatorname{Pr}\left\{\gamma_{5}<\hat{w}<\gamma_{6} \mid \tilde{\mathcal{H}}_{21}\right\}\right. \\
& +\operatorname{Pr}\left\{\gamma_{2}<\hat{w}<\gamma_{3} \mid \tilde{\mathcal{H}}_{22}\right\}+\operatorname{Pr}\left\{\gamma_{4}<\hat{w}<\gamma_{5} \mid \tilde{\mathcal{H}}_{22}\right\} \\
& +\operatorname{Pr}\left\{\begin{array}{c}
\left.\gamma_{3}<\hat{w}<\gamma_{4} \mid \tilde{\mathcal{H}}_{23}\right\} \\
\left(P_{23}\right)
\end{array}\right]
\end{aligned}
$$

where $\gamma_{1}, \gamma_{2}, \gamma_{3}, \gamma_{4}, \gamma_{5}$ and $\gamma_{6}$ are $4.5 w_{c}-w_{s}, 4 w_{c}-1.25 w_{s}$, $4 w_{c}-0.25 w_{s}, 4 w_{c}+0.25 w_{s}, 4 w_{c}+1.25 w_{s}$ and $4.5 w_{c}+w_{s}$ respectively the thresholds for circular constellation schemes at IF level.

Now the third stage of hierarchical classification is applied to QPSK and OQPSK, i.e., $\{\underbrace{\mathcal{H}_{4}, \mathcal{H}_{5}}_{\tilde{\mathcal{H}}_{231} \tilde{\mathcal{H}}_{232}}\}$ respectively, where $\tilde{\mathcal{H}}_{231}$ and $\tilde{\mathcal{H}}_{232}$ are the hypothesis in the third stage of decision. The pdf of hypothesis for the third stage is denoted by $p\left(\hat{w}\left|\mathcal{H}_{i}\right|_{i=4,5}\right)$, and its derivation is given in Appendix. The probability of correct classification in the third stage is given as

$$
\begin{aligned}
P_{23}= & \operatorname{Pr}\left\{\gamma_{7}<\hat{w}<\gamma_{8} \mid \tilde{\mathcal{H}}_{231}\right\} \\
& +\operatorname{Pr}\left\{\begin{array}{c}
\left.\gamma_{7}<\hat{w}<\gamma_{8} \mid \tilde{\mathcal{H}}_{232}\right\}, \\
\text { (No peak) }
\end{array}\right.
\end{aligned}
$$

where $\gamma_{7}$ and $\gamma_{8}$ are $0.5 w_{s}$ and $1.5 w_{s}$ respectively the thresholds for second order cyclic cumulant respectively. We now have all the probabilities and using (37) so we have $P_{c c}$ and the probability of error given as $P_{e}=1-P_{c c}$.

From the above studies, it is observed that all six modulations have their own distinct features so they can easily be classified by comparing the corresponding decision variables with the thresholds. The extensive simulation studies showed that the interval size can be minimized further for reducing the false alarm rate of classification in realistic scenarios.

Step 1: In macro classifier, BPSK and 16-QAM modulated signals can be classified by defining threshold values $\hat{c}_{[s, 4,2]}<T_{0}$ for BPSK modulated signal, $\hat{c}_{[s, 4,2]}>T_{1}$ for 16-QAM modulated signal and remaining window $T_{0} \leq \hat{c}_{[s, 4,2]} \leq T_{1}$ is for circularly modulated signals as shown in Fig. 1 and Fig. 8.

Step 2: The five intervals for $\tilde{\mathcal{H}}_{21}, \tilde{\mathcal{H}}_{22}$ and $\tilde{\mathcal{H}}_{23}$ given in Fig. 8 are optimized as follow:

The first and second search intervals for classifying MSK, i.e., for $\tilde{\mathcal{H}}_{21}$ modulated signal are given as

$$
\begin{aligned}
& {\left[\tilde{\gamma}_{1}, \tilde{\gamma}_{2}\right] \equiv\left[4 \hat{w}_{c}-2 w_{b w}, 4 \hat{w}_{c}-0.8 w_{b w}\right],} \\
& {\left[\tilde{\gamma}_{5}, \tilde{\gamma}_{6}\right] \equiv\left[4 \hat{w}_{c}+0.8 w_{b w}, 4 \hat{w}_{c}+2 w_{b w}\right],}
\end{aligned}
$$

where $w_{b w}$ is the $3 \mathrm{~dB}$ signal bandwidth, roughly estimated between $1.2 \hat{w}_{s} \leq w_{b w} \leq 2 \hat{w}_{s}$, and $\hat{w}_{s}$ is the estimated symbol rate frequency. The third and fourth search intervals for classifying 
$\pi / 4-\mathrm{QPSK}$, i.e., for $\tilde{\mathcal{H}}_{22}$ modulated signal are given as

$$
\begin{aligned}
& {\left[\hat{\gamma}_{2}, \hat{\gamma}_{3}\right] \equiv\left[4 \hat{w}_{c}-1.2 w_{b w}, 4 \hat{w}_{c}-0.2 w_{b w}\right],} \\
& {\left[\hat{\gamma}_{4}, \hat{\gamma}_{5}\right] \equiv\left[4 \hat{w}_{c}+0.2 w_{b w}, 4 \hat{w}_{c}+1.2 w_{b w}\right],}
\end{aligned}
$$

The fifth search interval for classifying QPSK and OQPSK, i.e., for $\tilde{\mathcal{H}}_{23}$ modulated signal is given as

$$
\left[\bar{\gamma}_{3}, \bar{\gamma}_{4}\right] \equiv\left[4 \hat{w}_{c}-0.05 \hat{w}_{c}, 4 \hat{w}_{c}+0.05 \hat{w}_{c}\right],
$$

where $\hat{w}_{c}$ is the estimated carrier frequency.

Step 3: At the second stage of classification, micro classifier classifies MSK, $\pi / 4-\mathrm{QPSK}$, and (QPSK, OQPSK). For QPSK and OQPSK further classification is required.

Step 4: For classifying QPSK and OQPSK one search interval is required, i.e.,

$$
\left[\tilde{\gamma}_{7}, \tilde{\gamma}_{8}\right] \equiv\left[w_{b w} / 2, w_{b w} / 1.2\right] .
$$

For classifying QPSK and OQPSK, a second order cyclic cumulant of baseband signal is obtained and if the cyclic cumulant peak is present at the symbol rate frequency, we classify the signal as QPSK, i.e., for $\tilde{\mathcal{H}}_{231}$ modulated signal otherwise as OQPSK, i.e., for $\tilde{\mathcal{H}}_{232}$ modulated signal as shown in Fig. 6 and Fig. 7.

\section{IMPLEMENTATION AND MEASUREMENT SetuP}

The measurements have been performed in Signal Processing for Wireless Communication Laboratory, IIT Patna. Configuration of the testbed is done by the software programmable NI PXIe platform [1], [2], [35]. In the transmitter, modulated signals BPSK, QPSK, OQPSK, $\pi / 4-\mathrm{QPSK}, \mathrm{MSK}$, and 16-QAM are generated first by using NI RF signal generator and fed into arbitrary function generator and then to LO and RF up-converter to transmit the signal through the air.

Signal Acquisition: In the RF search process, a specified RF filter span is selected first to receive the signal within this band. The received RF energy is then compared with a predefined threshold value. The predefined threshold is acquired through a calibration process where the energy of the received signal is calculated in the absence of transmitting signal. If the received signal energy is above the threshold value, the position of the peak is estimated, which is the estimated RF carrier frequency $\hat{w}_{c}$. Alternatively, the next burst signal is captured and the same process is repeated. The RF signal is down converted to analog IF signal by setting the LO frequency as $\hat{f}_{c}-5 \mathrm{MHz}\left(\hat{f}_{c}=\right.$ $\left.\hat{w}_{c} / 2 \pi\right)$. The proposed method needs a sampling rate of eight times the IF carrier to estimate the IF carrier without much ambiguity. Thus, for an $5 \mathrm{MHz}$ tIF carrier signal, minimum of $48 \mathrm{MHz}$ sampling rate is required to convert analog IF to digitized IF signal for a successful IF estimation.

IF and Baseband Signal Processing: The received digitized IF signal is passed to the MATLAB script, which runs inside the LabVIEW environment. The received real digitized IF signal is first converted to complex digitized IF signal, then IF carrier and signal bandwidth are estimated from PSD of the complex IF signal. Modulation classification is performed both at IF and baseband level to classify six-class of modulation schemes.

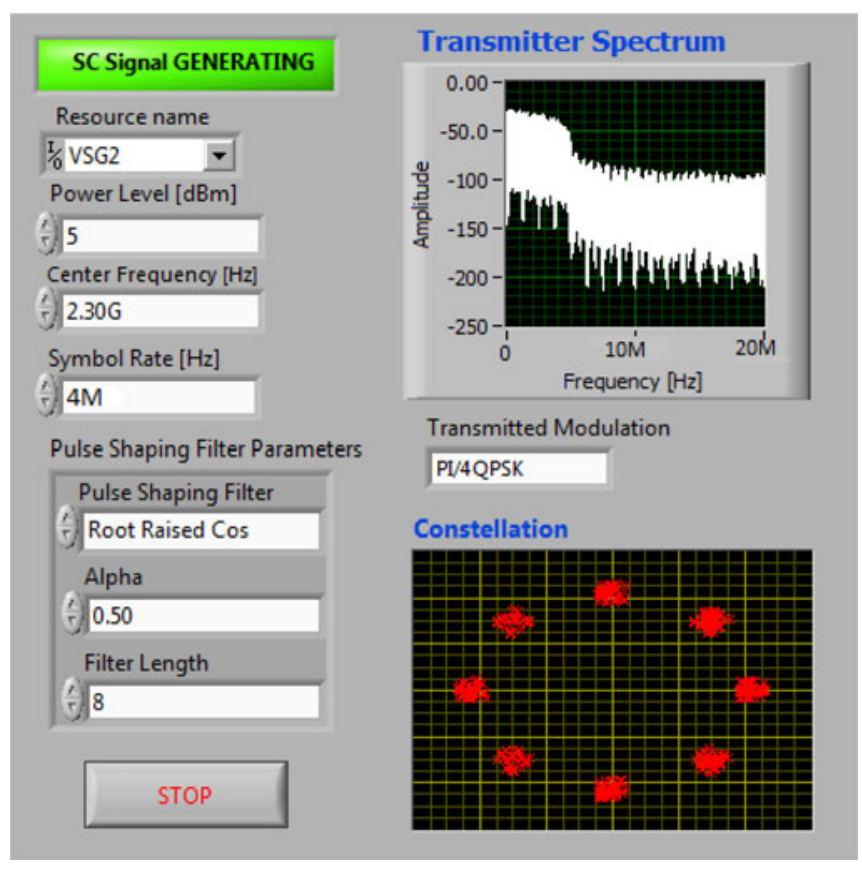

Fig. 9. Front panel of transmitter setup using PXIe-5673.

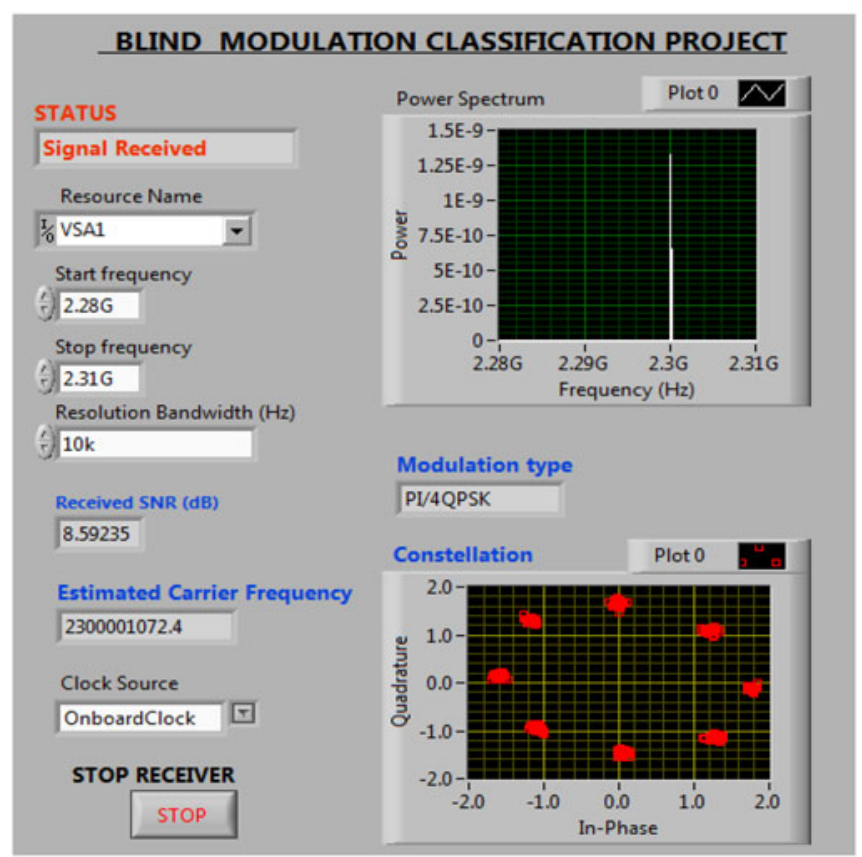

Fig. 10. Front panel of receiver setup using PXIe-5663.

Fig. 9 shows the front panel of transmitter setup using PXIe5673 and Fig. 10 shows the front panel of receiver setup using PXIe-5663. Fig. 11 shows the transmitter and receiver setup for $3 \mathrm{~m}$ distance.

\section{Simulation and Measurement Results}

In this section, the performance of BMC is evaluated through simulation and measurement results. The simulation and measurement parameters are provided in Table II. In the implemen- 


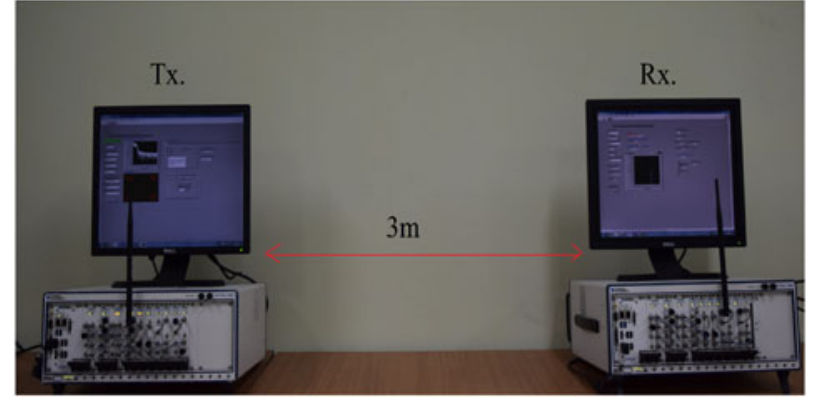

Fig. 11. Transmitter and receiver setup in signal processing for wireless communication laboratory at IIT Patna.

TABLE II

SiMULATION AND EXPERIMENT PARAMETERS OF SYSTEM

\begin{tabular}{lc}
\hline \hline Simulation parameters & Value \\
\hline Receiver sampling rate $F_{s}$ & $48 \mathrm{MHz}$ \\
Oversampling factor P & 12 \\
Roll off factor $\alpha$ & 0.5 \\
Carrier frequency $f_{c}$ & $5 \mathrm{MHz}$ \\
Symbol rate $f_{s}$ & $4 \mathrm{MHz}$ \\
Number of symbol L & 500 \\
Number of iteration & 1000 \\
Experimental parameters & \\
Receiver sampling rate $F_{s}$ & $48 \mathrm{MHz}$ \\
RF carrier frequency $f_{c}$ & $2.4 \mathrm{GHz}$ \\
IF & $5 \mathrm{MHz}$ \\
\hline \hline
\end{tabular}

tation process, we use $5 \mathrm{MHz}$ IF carrier frequency and $4 \mathrm{MHz}$ symbol rate. However, the IF carrier can be varied between 1 $\mathrm{MHz}$ to $5 \mathrm{MHz}$ and symbol rate can be varied between $100 \mathrm{kHz}$ to $5 \mathrm{MHz}$. We have implemented the testbed for six modulation schemes which are from linearly modulated constellations. The number of symbols for each iteration is set to 500 and the performance is measured for 1000 iterations.

Figs. 12 and 13 show that the success rate for measurement (M) and simulation (S) results for a six-class of modulation classification problem. It can be noticed that the measurement demonstrates better results than the results obtained by simulation. The fading involved in the simulation and the measurement may not be the same as the former is generated by assumption of Rayleigh fading and the latter one is mixed from the real channel during the measurement. As in the measurement, the distance between transmitter and receiver was not more than $3 \mathrm{~m}$, we may not get the fading dip during the measurement, but in simulation fading dip can be experienced due to the Rayleigh fading assumption.

It has been observed from Fig. 13 the performance of the proposed BMC is almost the same for all the modulation schemes. The classification between BPSK and 16-QAM is performed well as they are classified by using EC, and it has been known that EC has good property of classifying the modulation format if the signals are not from the circular constellation. It provides $100 \%$ accuracy even at $7 \mathrm{~dB}$ SNR. The $\pi / 4$-QPSK and MSK have $7 \mathrm{~dB}$ lower performance than BPSK and 16-QAM at low SNR region due to the split of their RF energy to different

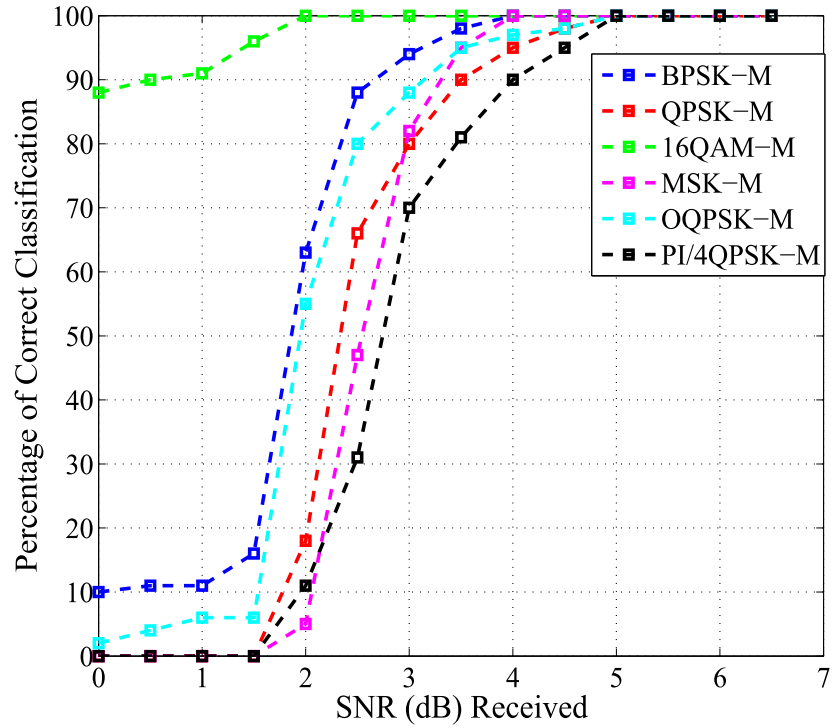

Fig. 12. Success rate for measurement (M) results for six-class of modulation classification problem.

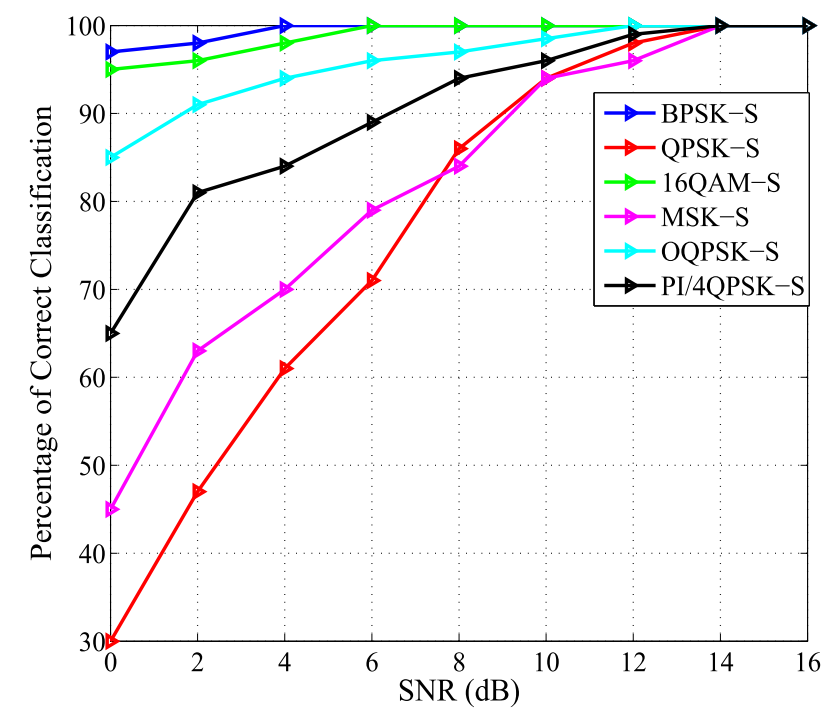

Fig. 13. Success rate for simulation (S) results for six- class of modulation classification problem.

locations. The $100 \%$ accuracy of $\pi / 4$-QPSK and MSK are observed at $14 \mathrm{~dB}$ SNR. The OQPSK and QPSK has also exhibited almost the same performance as $\pi / 4$-QPSK and MSK at low SNR region. It is because the symbol rate peak does not provide the global maximum at low SNR values and for a small roll-off factor of RRC filter.

Figs. 14 and 15 show the simulation result comparison of proposed classifier with EC and ML methods for flat fading channel. The proposed BMC method shows 100\% accuracy beyond $14 \mathrm{~dB}$ SNR. However, EC and ML method still provides some error even after $16 \mathrm{~dB}$ SNR. Moreover, EC and ML methods cannot work for all six-class modulation schemes and ML is not a blind method. The computational complexity of the proposed algorithm is of $\mathcal{O}(N \log N)$. The computational complexity of EC and maximum likelihood (ML) algorithm is 


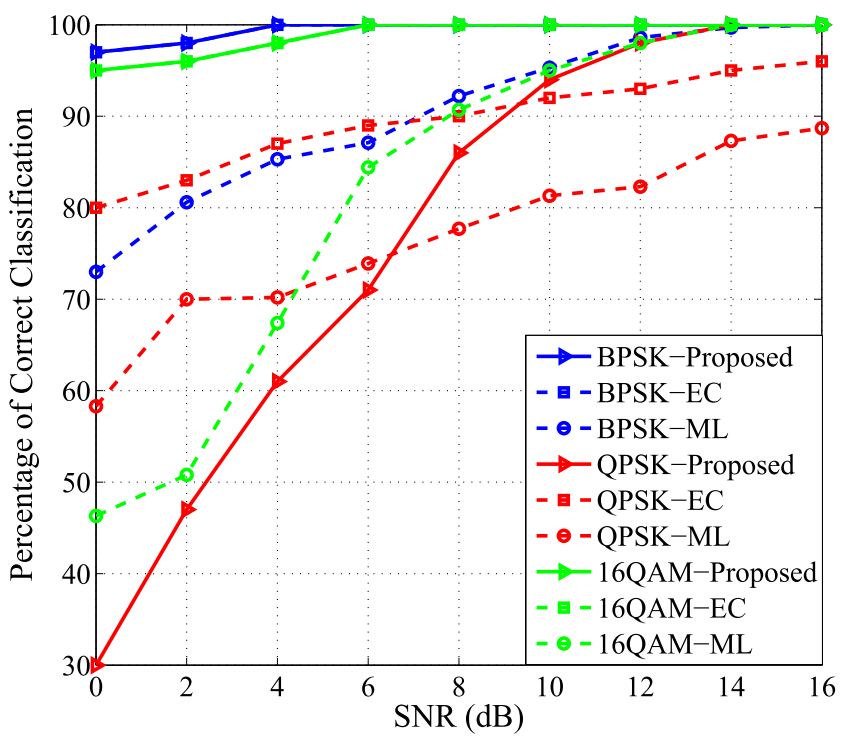

Fig. 14. Success rate for simulation result comparison of BPSK, QPSK and 16-QAM modulation schemes for proposed, elementary cumulant (EC) and maximum likelihood (ML) methods for flat fading channel.

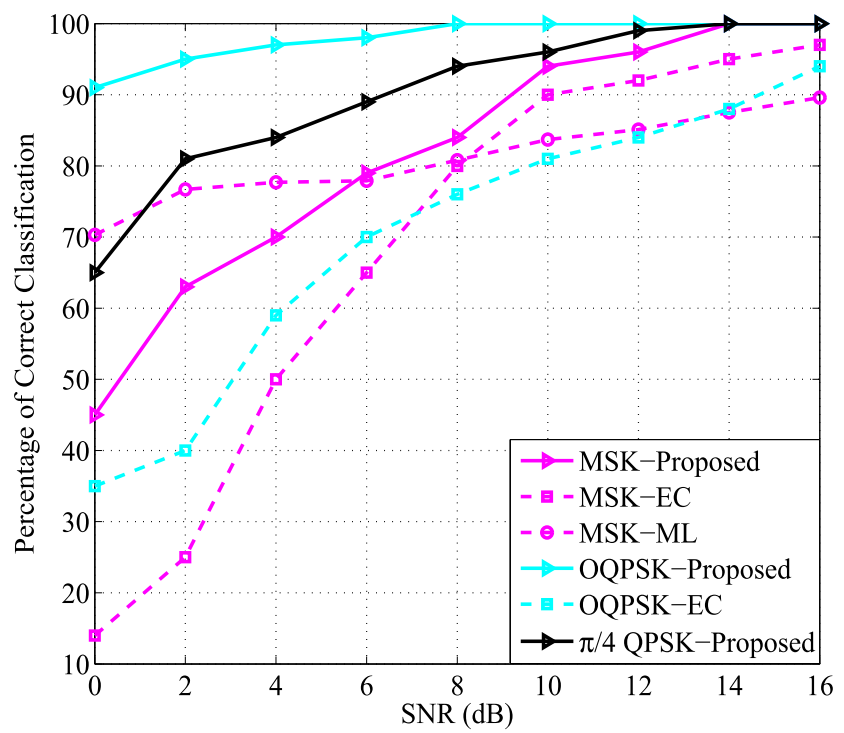

Fig. 15. Success rate for simulation result comparison of MSK, OQPSK and $\pi / 4$ QPSK modulation schemes for proposed, elementary cumulant (EC) and maximum likelihood (ML) methods for flat fading channel.

of $\mathcal{O}(N)$ and $\mathcal{O}\left(N \times M_{i} \times I\right)$ respectively, where $\mathrm{N}$ is the number of data samples, $M_{i}$ is the constellation size of the ith modulation scheme and $I$ is the total number of modulation schemes used for classification.

\section{CONCLUSION}

A new BMC has been proposed for classifying the modulation format for six different modulated signals namely BPSK, QPSK, OQPSK, $\pi / 4$-QPSK, MSK, and 16-QAM. The proposed method is based on the combination of $\mathrm{EC}$ and cyclic cumulant. The fourth order cyclic cumulant properties of $\pi / 4-\mathrm{QPSK}$ and MSK modulated signals are investigated as well as the second order cyclic cumulant properties of QPSK and OQPSK modulated signals. The performance of the proposed method derived through feature-based hierarchical hypothesis test is compared with ML and EC methods over a flat fading channels. However, EC and ML method still provide some error even at high SNR. Moreover, EC and ML methods cannot work for all six-class modulation schemes as discussed. The proposed BMC method has been implemented over a NI testbed, the performances are measured over an indoor environment and compared to the simulated results. It has been observed that the proposed hierarchical hypothesis test and feature-based BMC approach is robust in the presence of synchronization and phase errors. It also has better classification performance over a Rayleigh flat fading channel compared with other existing methods.

\section{APPENDIX}

From the fourth order EC given in (11), we define $\bar{c}_{[s, 4,2]}=$ $X[m]=\frac{1}{N} \sum_{n=1}^{N}|s[n]|^{4}$ joint pdf of $\mathrm{M}$ iid random variables $X[1], X[2], \ldots, X[M]$ is given as

$$
\left.p\left(\bar{c}_{[s, 4,2]}\right] \tilde{\mathcal{H}}_{i}\right)=c_{1} \cdot \exp \left[\frac{-1}{2 \sigma^{2}} \sum_{m=0}^{M-1} Y_{1}[m]\right] \times \prod_{m=0}^{M-1} Z_{1}[m],
$$

where $c_{1}=\frac{1}{2^{M}\left(2 \pi \sigma_{i}^{2}\right)^{M / 2}}, Y_{1}[m]=\left(\bar{c}_{[s, 4,2]}^{1 / 4}-\mu_{i}\right)^{2}$, and $Z_{1}[m]=$ $\bar{c}_{[s, 4,2]}^{-3 / 4}$.

For simplicity, we take (11) as $\hat{c}_{[s, 4,2]}=X[m]-Y[m]-$ $Z[m]$ where, $Y[m]=\left|\hat{c}_{[s, 2,0]}\right|^{2}$ and $Z[m]=2 \hat{c}_{[s, 2,1]}^{2}$. We want to find the pdf of $\hat{c}_{[s, 4,2]}$. This requires defining other transformations as $c_{[s, 4,2]}^{\prime}$ and $c_{[s, 4,2]}^{\prime \prime}$ given as $c_{[s, 4,2]}^{\prime}=Y[m]$ and $c_{[s, 4,2]}^{\prime \prime}=Z[m]$ respectively. As $X[m], Y[m]$ and $Z[m]$ are dependent random variables with pdf given in (40) its joint distribution is given as $p_{X, Y, Z}(x, y, z)$. After the inverse of $\hat{c}_{[s, 4,2]}$, $c_{[s, 4,2]}^{\prime}$ and $c_{[s, 4,2]}^{\prime \prime}$ the joint pdf $g\left(\hat{c}_{[s, 4,2]}, c_{[s, 4,2]}^{\prime}, c_{[s, 4,2]}^{\prime \prime}\right)=$ $p\left(\hat{c}_{[s, 4,2]}+c_{[s, 4,2]}^{\prime}+c_{[s, 4,2]}^{\prime \prime}, c_{[s, 4,2]}^{\prime}, c_{[s, 4,2]}^{\prime \prime}\right)$. Now we can find the marginal pdf of $\hat{c}_{[s, 4,2]}$ as

$$
\begin{aligned}
\left.g\left(\hat{c}_{[s, 4,2]}\right] \tilde{\mathcal{H}}_{i}\right)= & \int_{c_{[s, 4,2]}^{\prime \prime}} \int_{c_{[s, 4,2]}^{\prime}} p\left(\hat{c}_{[s, 4,2]}+c_{[s, 4,2]}^{\prime}\right. \\
& \left.+c_{[s, 4,2]}^{\prime \prime}, c_{[s, 4,2]}^{\prime}, c_{[s, 4,2]}^{\prime \prime}\right) d c_{[s, 4,2]}^{\prime} d c_{[s, 4,2]}^{\prime \prime} .
\end{aligned}
$$

Similarly as (40) we can find the pdf of the fourth order correlation function of received signal $\left.\tilde{y}_{i}[n]\right|_{i=2,3,4,5}$ as $p\left(c_{r[\tilde{y}, 4,0]}[n ; \mathbf{0}]\right)$. Using (15), (24) and (30), we first take the DFT which is given as

$$
\mathcal{F}\left(C_{[\tilde{y}, 4,0]}[\alpha ; \mathbf{0}]\right)=\frac{1}{N} \sum_{n=1}^{N} p\left(c_{r[\tilde{y}, 4,0]}[n ; \mathbf{0}]\right) e^{-j \alpha n} .
$$

Now by using [36] we can find the pdf, $p\left(\hat{w}\left|\tilde{\mathcal{H}}_{i j}\right|_{i j=21,22,23}\right)$, as the pdf of $\arg \max \left\{\mathcal{F}\left(C_{[\tilde{y}, 4,0]}[\alpha ; \mathbf{0}]\right)\right\}$. 
For the second order EC $\hat{c}_{[s, 2,1]}$ pdf is given as

$$
\begin{aligned}
p\left(\hat{c}_{[s, 2,1]}\left|\tilde{\mathcal{H}}_{i j k}\right|_{i j k=231,232}\right)= & c_{2} \cdot \exp \left[\frac{-1}{2 \sigma^{2}} \sum_{m=0}^{M-1} Y_{2}[m]\right] \\
& \times \prod_{m=0}^{M-1} Z_{2}[m]
\end{aligned}
$$

where $\quad c_{2}=\frac{1}{\left(2 \pi \sigma_{i j k}^{2}\right)^{M / 2}}, \quad Y_{2}[m]=\left(\hat{c}_{[s, 2,1]_{i j k}}^{1 / 2}-\mu_{i j k}\right)^{2}, \quad$ and $Z_{2}[m]=\hat{c}_{[s, 2,1]_{i j k}}^{-1 / 2}$.

Similarly, we can find the pdf, $p\left(\hat{w} \mid \tilde{\mathcal{H}}_{i j k}\right)$, using (34) as the pdf of $\arg \max \left\{\mathcal{F}\left(\hat{C}_{[s, 2,1]}[\alpha ; \mathbf{0}]\right)\right\}$.

\section{REFERENCES}

[1] S. Majhi and T. S. Ho, "Blind symbol-rate estimation and test bed implementation of linearly modulated signals," IEEE Trans. Veh. Technol., vol. 64, no. 3, pp. 954-963, Mar. 2015.

[2] S. Majhi, M. Kumar, and W. Xiang, "Implementation and measurement of blind wireless receiver for single carrier systems," IEEE Trans. Instrum. Meas., vol. 66, no. 8, pp. 1965-1975, Aug. 2017.

[3] J. Xiao, R. Hu, Y. Qian, L. Gong, and B. Wang, "Expanding LTE network spectrum with cognitive radios: From concept to implementation," IEEE Wireless Commun., vol. 20, no. 2, pp. 12-19, Apr. 2013.

[4] X. Wang, H.-C. Wu, S. Y. Chang, Y. Wu, and J.-Y. Chouinard, "Efficient non-pilot-aided channel length estimation for digital broadcasting receivers," IEEE Trans. Broadcast., vol. 55, no. 3, pp. 633-641, Sep. 2009.

[5] S. Majhi, B. J. Prakash, and M. Kumar, "Blind wireless receiver performance for single carrier systems," in Proc. Int. Conf. Commun. Signal Process., Apr. 2015, pp. 0521-0525.

[6] M. Kumar and S. Majhi, "Blind synchronization of OFDM system and CRLB derivation of CFO over fading channels," in Proc. 10th Int. Conf. Inform. Commun. Signal Process., Dec. 2015, pp. 1-6.

[7] S. Majhi and W. Xiang, "Blind symbol rate estimation and testbed implementation for linearly modulated signals," in Proc. IEEE 78th Veh. Technol. Conf., Sep. 2013, pp. 1-5.

[8] W. Saad, M. Ismail, and R. Nordin, "Survey of adaptive modulation scheme in MIMO transmission," J. Commun., vol. 7, no. 12, pp. 873-884, Dec. 2012.

[9] W. Wei and J. M. Mendel, "Maximum-likelihood classification for digital amplitude-phase modulations," IEEE Trans. Commun., vol. 48, no. 2, pp. 189-193, Feb. 2000.

[10] V. Chavali and C. da Silva, "Classification of digital amplitude-phase modulated signals in time-correlated non-Gaussian channels," IEEE Trans. Commun., vol. 61, no. 6, pp. 2408-2419, Jun. 2013.

[11] Z. Zhijin and L. Tao, "A MPSK modulation classification method based on the maximum likelihood criterion," in Proc. 7th Int. Conf. Signal Process. Aug. 2004, vol. 2, pp. 1805-1808.

[12] O. Dobre, A. Abdi, Y. Bar-Ness, and W. Su, "Survey of automatic modulation classification techniques: Classical approaches and new trends," IET Commun., vol. 1, no. 2, pp. 137-156, Apr. 2007.

[13] H. Li, O. A. Dobre, Y. Bar-Ness, and W. Su, "Quasi-hybrid likelihood modulation classification with nonlinear carrier frequency offsets estimation using antenna arrays," in Proc. IEEE Military Commun. Conf., vol. 1, Oct. 2005 , pp. $570-575$

[14] Y. Jie, Y. Chenzhou, and Z. Yue, "Modulation classification based on spectrogram," J. Syst. Eng. Electron., vol. 16, no. 3, pp. 475-488, Aug. 2005.

[15] L. Hong, "Classification of BPSK and QPSK signals in fading environment using the ICA technique," in Pro. 37th Southeastern Symp. Syst. Theory, Mar. 2005, pp. 491-494.

[16] Z. Zhu and A. Nandi, "Blind digital modulation classification using minimum distance centroid estimator and non-parametric likelihood function," IEEE Trans. Wireless Commun., vol. 13, no. 8, pp. 4483-4494, Aug. 2014

[17] G. Phukan, P. Bora, A. Rajesh, and C. Ramesh, "Amplitude normalization in blind modulation classification," in Proc. Nat. Conf. Commun., Feb. 2012, pp. 1-5.

[18] F. Hameed, O. Dobre, and D. Popescu, "On the likelihood-based approach to modulation classification," IEEE Trans. Wireless Commun., vol. 8, no. 12 , pp. 5884-5892, Dec. 2009.
[19] J. Xu, W. Su, and M. Zhou, "Likelihood-ratio approaches to automatic modulation classification," IEEE Trans. Syst. Man, Cybern., Part C: Appl. Rev., vol. 41, no. 4, pp. 455-469, Jul. 2011.

[20] A. Swami and B. M. Sadler, "Hierarchical digital modulation classification using cumulants," IEEE Trans. Commun., vol. 48, no. 3, pp. 416-429, Mar. 2000.

[21] W. Su, "Feature space analysis of modulation classification using very high-order statistics," IEEE Commun. Lett., vol. 17, no. 9, pp. 1688-1691, Sep. 2013.

[22] E. Soltanmohammadi and M. Naraghi-Pour, "Blind modulation classification over fading channels using expectation-maximization," IEEE Commun. Lett., vol. 17, no. 9, pp. 1692-1695, Sep. 2013.

[23] V. Orlic and M. Dukic, "Automatic modulation classification algorithm using higher-order cumulants under real-world channel conditions," IEEE Commun. Lett., vol. 13, no. 12, pp. 917-919, Dec. 2009.

[24] H.-C. Wu, M. Saquib, and Z. Yun, "Novel automatic modulation classification using cumulant features for communications via multipath channels," IEEE Trans. Wireless Commun., vol. 7, no. 8, pp. 3098-3105, Aug. 2008.

[25] S. Majhi, R. Gupta, and W. Xiang, "Novel blind modulation classification of circular and linearly modulated signals using cyclic cumulant," in Proc. IEEE Int. Symp. Pers. Indoor Mobile Radio Commun., Jul. 2017, to be published.

[26] M. Oner and O. A. Dobre, "On the second-order cyclic statistics of signals in the presence of receiver impairments," IEEE Trans. Commun., vol. 59, no. 12 , pp. 3278-3284, Dec. 2011.

[27] V. Chaithanya and V. Reddy, "Blind modulation classification in the presence of carrier frequency offset," in Proc. Int. Conf. Signal Process. Commun., Jul. 2010, pp. 1-5.

[28] O. A. Dobre, M. Oner, S. Rajan, and R. Inkol, "Cyclostationarity-based robust algorithms for QAM signal identification," IEEE Commun. Lett., vol. 16, no. 1, pp. 12-15, Jan. 2012.

[29] O. Dobre, Y. Bar-Ness, and W. Su, "Higher-order cyclic cumulants for high order modulation classification," in Proc. IEEE Military Commun. Conf. Proc., Oct. 2003, vol. 1, pp. 112-117.

[30] S. Majhi, R. Gupta, and B. Jeevan, "A blind modulation classification method for linearly modulated signal over single carrier systems," Filed India Patent 201631001884, Jan. 19, 2016.

[31] S. Majhi and M. Kumar, "Blind wireless receiver testbed implementation for single carrier systems," Filed India Patent 1337/KOL/2014, Dec. 22 , 2014

[32] G. Giannakis and G. Zhou, "Harmonics in multiplicative and additive noise: Parameter estimation using cyclic statistics," IEEE Trans. Signal Process., vol. 43, no. 9, pp. 2217-2221, Sep. 1995.

[33] P. Ciblat, P. Loubaton, E. Serpedin, and G. Giannakis, "Asymptotic analysis of blind cyclic correlation-based symbol-rate estimators," IEEE Trans. Inf. Theory, vol. 48, no. 7, pp. 1922-1934, Jul. 2002.

[34] F. Xiong, Digital Modulation Techniques, 2nd ed. Norwood, MA, USA: Artech House, 2006.

[35] National Instruments PXI platform, National Instruments, Banglore, India, 2017. [Online]. Available: http://www.ni.com/pxi/

[36] B. L. S. P. Rao, A first Course in Probability and Statistics. Singapore: World Scientific, 2009 